\title{
S-folds and $4 \mathrm{~d} \mathcal{N}=3$ superconformal field theories
}

\author{
Ofer Aharony ${ }^{a}$ and Yuji Tachikawa ${ }^{b, c}$ \\ ${ }^{a}$ Department of Particle Physics and Astrophysics, \\ Weizmann Institute of Science, Rehovot 7610001, Israel \\ ${ }^{b}$ Department of Physics, Faculty of Science, \\ University of Tokyo, Bunkyo-ku, Tokyo 113-0033, Japan \\ ${ }^{c}$ Kavli Institute for the Physics and Mathematics of the Universe, \\ University of Tokyo, Kashiwa, Chiba 277-8583, Japan \\ E-mail: ofer.aharony@weizmann.ac.il, yuji.tachikawa@ipmu.jp
}

\begin{abstract}
S-folds are generalizations of orientifolds in type IIB string theory, such that the geometric identifications are accompanied by non-trivial S-duality transformations. They were recently used by García-Etxebarria and Regalado to provide the first construction of four dimensional $\mathcal{N}=3$ superconformal theories. In this note, we classify the different variants of these $\mathcal{N}=3$-preserving S-folds, distinguished by an analog of discrete torsion, using both a direct analysis of the different torsion classes and the compactification of the S-folds to three dimensional M-theory backgrounds. Upon adding D3-branes, these variants lead to different classes of $\mathcal{N}=3$ superconformal field theories. We also analyze the holographic duals of these theories, and in particular clarify the role of discrete gauge and global symmetries in holography.
\end{abstract}

Keywords: Extended Supersymmetry, Brane Dynamics in Gauge Theories, F-Theory, D-branes

ARXIV EPRINT: 1602.08638 


\section{Contents}

1 Introduction and summary of results 1

2 Preliminary analysis 3

2.1 Three general properties of $\mathcal{N}=3$ SCFTs 4

2.2 D3-branes on S-folds and their discrete symmetries 5

3 Holographic dual $\quad 8$

3.1 AdS duals of CFTs with discrete symmetries 8

$\begin{array}{lll}3.2 & \text { Discrete torsion } & 10\end{array}$

$\begin{array}{lll}3.3 & \text { Generalized Pfaffians } & 10\end{array}$

$\begin{array}{ll}3.4 & \text { Realization of the } \mathbb{Z}_{k} \text { gauge theory in F-theory } \\ \end{array}$

4 Comparison to M-theory $\quad 13$

4.1 Discrete fluxes in M-theory on $\left(\mathbb{C}^{3} \times T^{2}\right) / \mathbb{Z}_{k} \quad 13$

$\begin{array}{ll}4.2 \text { M2-brane and D3-brane charges } & 15\end{array}$

$\begin{array}{lll}4.2 .1 \quad k=2 & 16\end{array}$

$\begin{array}{lll}4.2 .2 \quad k=3 & 17\end{array}$

$\begin{array}{lll}4.2 .3 \quad k=4 & 18\end{array}$

$\begin{array}{lll}4.2 .4 k=6 & 19\end{array}$

5 Special cases and $\mathcal{N}=4$ enhancement $\quad 21$

\section{Introduction and summary of results}

Field theories with superconformal symmetry are useful laboratories for learning about the behavior of quantum field theories in general, and strongly coupled field theories in particular. This is because the superconformal symmetry allows many computations to be performed in these theories, using methods such as localization, integrability, and the superconformal bootstrap.

The field theories (above two dimensions) about which the most is known are $\mathcal{N}=$ 4 superconformal field theories (SCFTs) in four dimensions, that have been called the 'harmonic oscillator of quantum field theories'. These theories have an exactly marginal deformation, and it is believed that they are all gauge theories with some gauge group $G$, such that the exactly marginal deformation is the gauge coupling constant. In these theories many observables have already been computed as functions (trivial or non-trivial) of the coupling constant, and there is a hope that they can be completely solved.

Four dimensional theories with $\mathcal{N}=2$ superconformal symmetry have also been extensively studied. Some of these theories have exactly marginal deformations and corresponding weak coupling limits (at least for some sector of the theory), while others do not. 
We do not yet have a full classification of $\mathcal{N}=2$ SCFTs (for rank- 1 theories, see [1-3]), though a large class of theories, called class S, has been constructed following [4]. Many observables can be computed also in $\mathcal{N}=2$ SCFTs, at least if they have a weak coupling limit, they are connected by renormalization group flows to theories that have such limits, or they are one of the theories of class S.

Four dimensional $\mathcal{N}=3$ theories should naively provide an intermediate class of theories, that is more general than $\mathcal{N}=4$, but such that more computations can be made than in general $\mathcal{N}=2$ theories. $\mathcal{N}=3$ SCFTs (that are not also $\mathcal{N}=4$ SCFTs) have no exactly marginal deformations $[5,6]$ and thus no weak coupling limits that would aid in classifying and performing computations in these theories. Until recently no $\mathcal{N}=3$ SCFTs were known, but recently a class of such theories was constructed by García-Etxebarria and Regalado in [7]. Their construction uses a generalization of orientifolds in string theory.

Orientifold 3-planes (the generalization to other dimensions is straightforward) are defined in type IIB string theory as planes in space-time, such that in the transverse space $y \in \mathbb{R}^{6}$ to these orientifolds, there is an identification between the points $y$ and $(-y)$, but with opposite orientations for strings (or, equivalently, with an opposite value for the $B_{2}$ and $C_{2} 2$-form potentials of type IIB). This means that we identify configurations related by a $\mathbb{Z}_{2}$ symmetry that involves a spatial reflection in the transverse $\mathrm{SO}(6)$, and also a transformation $(-I)$ in the $\mathrm{SL}(2, \mathbb{Z})$ S-duality group of type IIB string theory. This breaks half of the supersymmetry, preserving a four dimensional $\mathcal{N}=4$ supersymmetry. In particular, putting $N$ D3-branes on the orientifold (these do not break any extra supersymmetries) gives at low energies four dimensional $\mathcal{N}=4$ SCFTs.

In [7] this was generalized to identifying configurations related by a $\mathbb{Z}_{k}$ symmetry, that acts both by a $(2 \pi / k)$ rotation in the three transverse coordinates in $\mathbb{C}^{3}=\mathbb{R}^{6}$, and by an element of $\operatorname{SL}(2, \mathbb{Z})$ whose $k^{\prime}$ th power is the identity. We will call the fixed planes of such transformations S-folds; ${ }^{1}$ for $k=2$ they are the same as the usual orientifolds. Viewing $\mathrm{SL}(2, \mathbb{Z})$ as the modular group of a torus, the $\mathbb{Z}_{k}$ S-duality transformation may be viewed as a rotation of the torus by an angle $(2 \pi / k)$; such a rotation maps the torus to itself if and only if $k=3,4,6$ and its modular parameter is $\tau=e^{2 i \pi / k}$, so S-folds of this type exist only for these values of $k$ and $\tau$. It is natural to define such an identification using F-theory [17], in which the $\mathrm{SL}(2, \mathbb{Z})$ S-duality group is described as adding an extra zero-size torus whose modular parameter is the coupling constant $\tau$ of type IIB string theory; in this language the S-folds of [7] are the same as F-theory on $\left(\mathbb{C}^{3} \times T^{2}\right) / \mathbb{Z}_{k}$. This specific $\mathbb{Z}_{k}$ identification preserves a four dimensional $\mathcal{N}=3$ supersymmetry. So, putting $N$ D3-branes on the S-fold gives at low energies theories with $\mathcal{N}=3$ superconformal symmetry. Note that D3-branes sitting on the S-fold are invariant under all the transformations discussed above, and, in particular, the D3-brane charge of the S-fold is well-defined.

In this note we analyze further the theories constructed in [7]. We focus on asking what are the extra parameters associated with S-folds, analogous to discrete torsion for

\footnotetext{
${ }^{1}$ This term, first coined in [8], generalizes the term T-folds that is used to describe identifications by elements of the T-duality group. S-folds of string theory involving S-duality twists together with shifts along a circle were studied, for instance, in [9-13], and similar S-folds were studied in the $4 \mathrm{~d} \mathcal{N}=4 \mathrm{SYM}$ theory in [14-16].
} 
orbifolds and orientifolds (namely, to non-trivial configurations of the various $p$-forms of type IIB string theory in the presence of the S-fold). S-folds with different parameters can carry different D3-brane charges. Upon putting $N$ D3-branes on the S-fold, these discrete parameters label different $\mathcal{N}=3$ SCFTs. Our main result is a classification of these extra parameters (following a preliminary discussion in [7]); we show that there are two variants of S-folds with $k=3,4$ and just a single variant with $k=6$. Each variant leads to different $\mathcal{N}=3$ SCFTs, with different central charges and chiral operators.

In some cases discrete global symmetries play an interesting role. In a specific type of orientifold (the $O 3^{-}$plane), the theory of $N$ D3-branes on the orientifold is an $\mathrm{O}(2 N)$ gauge theory, and this may be viewed as an $\mathrm{SO}(2 N)$ gauge theory in which a discrete global $\mathbb{Z}_{2}$ symmetry is gauged. Both the $\mathrm{SO}(2 N)$ and the $\mathrm{O}(2 N)$ theories exist as $\mathcal{N}=4$ SCFTs, and a few of their properties are different. We will see that a similar phenomenon happens also for D3-branes on S-folds, and discuss its realization in the AdS/CFT dual of these theories, in terms of discrete gauge symmetries in the bulk. This dual is obviously given by F-theory on $A d S_{5} \times\left(S^{5} \times T^{2}\right) / \mathbb{Z}_{k}$.

We begin in section 2 with a naive analysis of the possible discrete parameters, by considering the possible discrete identifications on the moduli space of $N$ D3-branes, and the effects of discrete symmetries. In section 3 we discuss the holographic duals of the $\mathcal{N}=3$ SCFTs, and the realization of the discrete symmetries there. We show that holography suggests that only some of the possibilities found in the naive analysis are consistent. The compactification of F-theory on $\left(\mathbb{C}^{3} \times T^{2}\right) / \mathbb{Z}_{k}$ on a circle gives M-theory on $\left(\mathbb{C}^{3} \times T^{2}\right) / \mathbb{Z}_{k}$, with each S-fold splitting into several $\mathbb{C}^{4} / \mathbb{Z}_{l}$ singularities in M-theory. In section 4 we show that the consistency of this reduction implies that indeed only the possibilities found in section 3 are consistent. In section 5 we discuss the fact that for specific values of $k$ and $N$ some variants of the $\mathcal{N}=3$ theories have enhanced $\mathcal{N}=4$ supersymmetry, with gauge groups $\mathrm{SU}(3), \mathrm{SO}(5)$ and $G_{2}$, and check the consistency of this. This provides new brane constructions and new (strongly coupled) AdS duals for these specific $\mathcal{N}=4$ SCFTs.

In this paper we discuss only the S-folds which give rise to four dimensional $\mathcal{N}=3$ supersymmetric theories. It would be interesting to study S-folds that preserve different amounts of supersymmetry, and that have different dimensions. A particularly interesting case, which should have many similarities to our discussion, is S-folds preserving four dimensional $\mathcal{N}=2$ supersymmetry. We leave the study of these theories to the future.

\section{Preliminary analysis}

In this paper we study the S-folds introduced by García-Etxebarria and Regalado in [7], which are equivalent to F-theory on $\left(\mathbb{C}^{3} \times T^{2}\right) / \mathbb{Z}_{k}$ for $k=3,4,6$, generalizing the standard orientifold 3-planes that arise for $k=2$. Just as the orientifold 3-planes have four variants, $\mathrm{O3}^{-}, \widetilde{O 3}^{-}, \mathrm{O3}^{+}$and $\widetilde{\mathrm{O3}}{ }^{+}$, that differ by discrete fluxes, we expect that these new S-folds could also have a few variants. In this section we perform a preliminary analysis of the possible variants, from the viewpoint of the moduli space of the $\mathcal{N}=3$ superconformal field theories realized on $N$ D3-branes probing these S-folds. In the following sections we will discuss additional constraints on the possible variants. 


\subsection{Three general properties of $\mathcal{N}=3$ SCFTs}

We start from the following three properties of theories of this type, that are generally believed to hold:

- The conformal anomalies (central charges) $a$ and $c$ are equal in any $\mathcal{N}=3$ SCFT [5]:

$$
a=c
$$

The value of $a=c$ determines also all anomalies of the $\mathrm{SU}(3)_{R} \times \mathrm{U}(1)_{R}$ symmetries in these theories.

- The geometry of the gravitational dual of $N$ D3-branes sitting on a singularity involving a $\mathbb{Z}_{k}$ identification of their transverse space, and carrying $\epsilon$ units of D3-brane charge, is $\mathrm{AdS}_{5} \times S^{5} / \mathbb{Z}_{k}$, with $(N+\epsilon)$ units of 5 -form flux. This implies that the large $N$ central charge of the corresponding CFTs is

$$
a \sim c \sim k(N+\epsilon)^{2} / 4+O\left(N^{0}\right) .
$$

- In any $\mathcal{N}=2$ theory, the Coulomb branch operators (chiral operators whose expectation values label the Coulomb branch of the moduli space, namely the space of vacuum expectation values of the scalars in the vector multiplets) form a ring generated by $n$ operators, whose expectation values are all independent without any relation. Furthermore, up to a caveat mentioned below, there is a relation

$$
2 a-c=\sum_{i=1}^{n}\left(2 \Delta_{i}-1\right) / 4
$$

where $\Delta_{i}$ are the scaling dimensions of the generators of the Coulomb branch operators.

The property (2.2) can be derived easily following the analysis of [18-20]. Essentially, the curvature of the AdS space is supported by the total energy of the five-form field, and the volume of the $\mathbb{Z}_{k}$ quotient is $1 / k$ of the volume before the quotient, changing the value of Newton's constant on $A d S_{5}$ by a factor of $k$.

The equation (2.3), originally conjectured in [21], was given a derivation that applies to a large subclass of $\mathcal{N}=2$ theories in [22] (though it is not clear that this subclass includes the theories we discuss here). More precisely, this relation applies only to gauge theories whose gauge group has no disconnected parts. To illustrate this, consider the $\mathcal{N}=4$ super Yang-Mills theories with gauge groups $\mathrm{U}(1)$ and $\mathrm{O}(2)$. They have the same central charges $2 a-c=1 / 4$, coming from a single free vector multiplet, but the gauge-invariant Coulomb branch operator has dimension 1 for the former and 2 for the latter, so that property 3 only holds for the former. This is because the scalars in the vector multiplet change sign under the disconnected component of the $\mathrm{O}(2)$ gauge group. The $\mathrm{O}(2)$ theory is obtained by gauging a $\mathbb{Z}_{2}$ global symmetry of the $\mathrm{U}(1)=\mathrm{SO}(2)$ theory, where the $\mathbb{Z}_{2}$ symmetry is generated by the quotient $\mathrm{O}(2) / \mathrm{SO}(2)$; this operation does not change the central charges. 
This means that the $\mathrm{O}(2)$ theory includes a $\mathbb{Z}_{2}$ gauge theory, implying that it has a nontrivial $\mathbb{Z}_{2} 2$-form global symmetry in the sense of [23], as we further discuss below. We assume in the following that the relation (2.3) holds whenever the theory does not have any non-trivial 2-form global symmetry, even if the theory does not have a gauge theory description.

\subsection{D3-branes on S-folds and their discrete symmetries}

Let us now see what the three properties recalled above tell us about the properties of S-folds. Let us probe the $\mathbb{Z}_{k}$ S-fold by $N$ D3-branes. The moduli space of $\mathcal{N}=3$ theories is described by the expectation values of scalars in $\mathcal{N}=3$ vector multiplets, which are identical to $\mathcal{N}=4$ vector multiplets; viewing an $\mathcal{N}=3$ theory as an $\mathcal{N}=2$ theory, these decompose into a vector multiplet and a hypermultiplet. Let us choose a specific $\mathcal{N}=2$ subgroup of the $\mathcal{N}=3$ symmetry, and consider the component of the moduli space which is a Coulomb branch from the point of view of this $\mathcal{N}=2$ subgroup. This implies that all the D3-branes lie on a particular $\mathbb{C} / \mathbb{Z}_{k}$ within $\mathbb{C}^{3} / \mathbb{Z}_{k}$. Denote by $z_{i}(i=1, \ldots, N)$ the positions on $\mathbb{C}$ of these D3-branes. Since the D3-branes are identical objects, the identifications that are imposed on the moduli space by the geometry are

$$
\begin{aligned}
& z_{i} \leftrightarrow z_{j}, \quad \text { all other } z_{a} \text { fixed, } \\
& z_{i} \mapsto \gamma z_{i}, \quad \text { all other } z_{j} \text { fixed, }
\end{aligned}
$$

where $\gamma \equiv \exp (2 \pi \mathrm{i} / k)$. For the purpose of the moduli space identifications we can ignore the additional S-duality transformation.

From these identifications we can see that the gauge-invariant Coulomb branch operators are the symmetric polynomials of $z_{i}{ }^{k}$, and their generators are $\left(\sum_{i=1}^{N} z_{i}{ }^{j k}\right)$ for $j=1, \cdots, N$, with dimensions

$$
k, 2 k, \cdots, N k \text {. }
$$

From (2.1) and (2.3), this naively implies that

$$
4 a=4 c=k N^{2}+(k-1) N .
$$

Applying (2.2), we find that the D3-brane charge of the $\mathbb{Z}_{k}$ S-fold is $\epsilon=(k-1) /(2 k)$.

As discussed above, we know that even for $k=2$ this is not the whole story, due to the possibility of discrete torsion and discrete symmetries. Let us review in detail this $k=2$ case, in a language that will be useful for our later analysis. Among the known O3-planes, the above analysis only applies to $\widetilde{O 3^{-}}, O 3^{+}$and $\widetilde{O 3^{+}}$, for which $\epsilon=(k-1) /(2 k)=+1 / 4$ is the correct value. When the orientifold is $O 3^{-}$, the $\mathrm{O}(2 N)$ gauge group arising on $N$ D3branes can be viewed as a $\mathbb{Z}_{2}$ gauging of the $\mathrm{SO}(2 N)$ gauge theory, and thus, to compute $a$ and $c$, we need to use the $\mathrm{SO}(2 N)$ theory instead of the $\mathrm{O}(2 N)$ theory. In the $\mathrm{SO}(2 N)$ theory the residual gauge symmetries on the Coulomb branch, acting on the eigenvalues of a matrix in the adjoint representation of $\mathrm{SO}(2 N)$, are generated by

$$
\begin{aligned}
z_{i} & \leftrightarrow z_{j}, & & \text { all other } z_{a} \text { fixed } \\
\left(z_{i}, z_{j}\right) & \mapsto\left(-z_{i},-z_{j}\right), & & \text { all other } z_{a} \text { fixed }
\end{aligned}
$$


The dimensions of the independent gauge-invariant operators are then

$$
2,4, \cdots, 2(N-1) ; N
$$

where the last invariant is the Coulomb branch expression for the Pfaffian of an adjoint matrix of $\mathrm{SO}(2 N), z_{1} z_{2} \ldots z_{N}$. From this list we can compute $a$ and $c$ again using (2.1) and $(2.3)$ and find

$$
4 a=4 c=2 N^{2}-N,
$$

from which we find $\epsilon=-1 / 4$ from (2.2). This is indeed the correct value for $\mathrm{O3}^{-}$.

The $\mathrm{SO}(2 N)$ theory has a discrete $\mathbb{Z}_{2}$ global symmetry, corresponding to gauge transformations by elements of $\mathrm{O}(2 N)$ which are not elements of $\mathrm{SO}(2 N)$. Note that in the $\mathrm{SO}(2 N)$ theory these are global symmetries rather than gauge symmetries. On the moduli space one of these transformations acts as $z_{1} \rightarrow-z_{1}$, and with this extra identification the group generated by (2.7) becomes the same as the group (2.4) acting on the D3-branes. In particular, this identification projects out the Pfaffian operator of dimension $N$, such that after it we obtain the Coulomb branch operators with dimensions (2.5). So, for this specific orientifold plane, the theory on the D3-branes is described by a $\mathbb{Z}_{2}$ quotient of some 'parent' theory which has a different group of identifications (2.7), and correspondingly different Coulomb branch operators. Only after gauging a $\mathbb{Z}_{2}$ global symmetry of this 'parent' theory do we get the theory of the D3-branes on the $O 3^{-}$orientifold. We stress here that this gauging of $\mathbb{Z}_{2}$ symmetry does not change the central charges.

It is natural to ask how we could know directly from the theory of the D3-branes on the $\mathrm{O}^{-}$plane that its central charges are not the same as the naive ones, because it arises as a $\mathbb{Z}_{2}$ gauging of some other theory. As discussed in [23, 24], when we have a discrete $\mathbb{Z}_{p}$ global symmetry, we have local operators that transform under this symmetry, as well as 3 -plane operators that describe domain walls separating vacua that differ by a $\mathbb{Z}_{p}$ transformation. When we gauge the $\mathbb{Z}_{p}$ global symmetry, these local and 3-plane operators disappear from the spectrum. Instead we obtain new 2-plane operators (that may be viewed as worldvolumes of strings), characterized by having a $\mathbb{Z}_{p}$ gauge transformation when we go around them. These 2 -plane operators are charged under a 2 -form $\mathbb{Z}_{p}$ global symmetry in the language of [23]. So whenever we have a theory with a 2 -form $\mathbb{Z}_{p}$ global symmetry, it is natural to expect that it arises by gauging a $\mathbb{Z}_{p}$ global symmetry of some 'parent' theory. And indeed, the analysis of [23] implies that this 'parent' theory can be obtained by gauging the 2 -form $\mathbb{Z}_{p}$ global symmetry. Thus, whenever we have a theory with a $\mathbb{Z}_{p}$ 2 -form global symmetry, we expect that its central charges would not be given by (2.3), but rather by those of its 'parent' theory. ${ }^{2}$

Suggested by this analysis for $k=2$, we expect that also for $k=3,4,6$, different versions of S-folds will be characterized by different 2 -form $\mathbb{Z}_{p} \subset \mathbb{Z}_{k}$ global symmetries for the corresponding theories on the D3-branes, that will imply that these theories arise from 'parent' theories with $\mathbb{Z}_{p}$ global symmetries. The analysis above suggests that the identifications on the moduli spaces of these 'parent' theories should be subgroups of the

\footnotetext{
${ }^{2}$ Gauging a discrete $\mathbb{Z}_{p}$ global symmetry does not change the dynamics on $\mathbb{R}^{4}$, but it does change the spectrum of local and non-local operators.
} 
group generated by (2.4), such that the ring of invariants is polynomial without any relation, and such that adding an extra $\mathbb{Z}_{p}$ generator produces the group (2.4). Luckily such groups are already classified and are known as Shephard-Todd complex reflection groups; the fact that the ring of invariants is polynomial without any relation if and only if the group acting on $\mathbb{C}^{N}$ is a complex reflection group is known as the theorem of Chevalley, Shephard and Todd. ${ }^{3}$ For more on complex reflection groups, see [26].

In our case, the available groups are known as $G(N, p, k)$, and are generated by the following elements:

$$
\begin{aligned}
z_{i} & \leftrightarrow z_{j}, & & \text { all other } z_{a} \text { fixed, } \\
\left(z_{i}, z_{j}\right) & \mapsto\left(\gamma z_{i}, \gamma^{-1} z_{j}\right), & & \text { all other } z_{a} \text { fixed, } \\
z_{i} & \mapsto \gamma^{p} z_{i}, & & \text { all other } z_{j} \text { fixed }
\end{aligned}
$$

where $p$ is a divisor of $k$. By adding another $\mathbb{Z}_{p}$ generator acting as $z_{i} \mapsto \gamma z_{i}$, all these groups become the groups (2.4). Denoting $\ell=k / p$, we see that if there is a 'parent' theory with the identifications (2.10) on its moduli space, then its gauge-invariant Coulomb branch operators would be generated by the symmetric polynomials of $z_{i}^{k}$ and by $\left(z_{1} z_{2} \cdots z_{N}\right)^{\ell}$, and therefore the dimensions of the Coulomb branch generators would be given by

$$
k, 2 k, \ldots,(N-1) k ; N \ell .
$$

From (2.1) and (2.3) we then find that the central charges of the 'parent' theory, and also of its $\mathbb{Z}_{p}$ quotient that would describe the theory of $N$ D3-branes on the corresponding S-fold, are given by

$$
4 a=4 c=k N^{2}+(2 \ell-k-1) N .
$$

Note that even though the $\mathbb{Z}_{p}$-gaugings of the theories with different values of $\ell$ all have the same moduli space, they are distinct theories with different central charges. When $p$ is not prime, one can also gauge subgroups of $\mathbb{Z}_{p}$, giving rise to additional theories, which again are not equivalent to the theories for other values of $\ell$ (even though they may have the same moduli space).

Therefore, it seems at this stage, that each $\mathbb{Z}_{k}$ S-fold with $k=2,3,4,6$ can have variants labeled by $\ell$ which is an integer dividing $k$. The D3-brane charges $\epsilon_{k, \ell}$ of these S-folds can be easily found using (2.2) and (2.12):

$$
\begin{array}{|l|l|l|l|l|l|}
\hline & \ell=1 & \ell=2 & \ell=3 & \ell=4 & \ell=6 \\
\hline k=2 & -1 / 4 & +1 / 4 & & & \\
k=3 & -1 / 3 & & +1 / 3 & & \\
k=4 & -3 / 8 & -1 / 8 & & +3 / 8 & \\
k=6 & -5 / 12 & -1 / 4 & -1 / 12 & & +5 / 12 \\
\hline
\end{array}
$$

Note that just from the analysis above it is far from clear that $\mathrm{S}$-folds and $\mathcal{N}=3$ theories corresponding to such identifications actually exist. However, we expect any Sfold to fall into one of these categories. In the next two sections, we will see that the

\footnotetext{
${ }^{3}$ See $[25]$ for a recent use of Shephard-Todd complex reflection groups in four-dimensional $\mathcal{N}=2$ theories.
} 
S-fold variants that really exist are only those shaded in the table (2.13) above, first by carefully studying the holographic dual and then by comparing with M-theory. Note also that there could be more than one theory with the same identifications. For $k=2$ there are three orientifold-types with the same $\ell=2$ identifications, though they all give rise to the same theory on D3-branes because they are all related by S-duality. We do not know the corresponding situation in our case. We also cannot rule out the existence of $\mathcal{N}=3$ theories having Coulomb branch operators (2.11) also for the values of $\ell$ and $k$ that do not come from S-folds. It would be interesting to shed further light on these questions, perhaps by a conformal bootstrap analysis of $\mathcal{N}=3 \mathrm{SCFTs}$, or by analyzing the two dimensional chiral rings of the corresponding theories $[27,28]$.

\section{Holographic dual}

\subsection{AdS duals of CFTs with discrete symmetries}

Theories of quantum gravity are not expected to have any global symmetries (see, for instance, [24]). There are very strong arguments that this is the case for continuous symmetries, and it is believed to be true also for discrete symmetries. In the AdS/CFT correspondence, this implies that any global symmetry in the CFT should come from a gauge symmetry in anti-de Sitter (AdS) space.

For continuous global symmetries it is known that indeed they come from gauge fields in the bulk, with a boundary condition that sets their field strength to zero on the boundary. In some cases (like $\mathrm{AdS}_{4}[29]$ ) there is also another consistent boundary condition in which the field strength does not go to zero on the boundary, and its boundary value becomes a gauge field in the CFT. Changing the boundary condition may be interpreted as gauging the global symmetry in the CFT (in cases where this gauging still gives a CFT).

In this section we discuss the analogous statements for discrete symmetries. We focus on the $A d S_{5} \leftrightarrow \mathrm{CFT}_{4}$ case, but the generalization to other dimensions is straightforward. The discussion here is a special case of the discussion in appendix B of [23], generalized to five dimensions, but as far as we know its implications for the AdS/CFT correspondence were not explicitly written down before (see also [30]).

Consider a $\mathbb{Z}_{k}$ gauge symmetry on $A d S_{5}$ (thought of as a sector of a full theory of quantum gravity on $A d S_{5}$, that is dual to a $4 \mathrm{~d}$ CFT). A universal way to describe such a gauge symmetry in five space-time dimensions is by a topological theory of a 1 -form $A$ and a 3 -form $C$, with an action

$$
\mathcal{L}=\frac{i k}{2 \pi} A \wedge d C
$$

$A$ can be thought of as the gauge field for a $\mathbb{Z}_{k}$ symmetry; for example, the action above arises from a $\mathrm{U}(1)$ gauge symmetry that is spontaneously broken to $\mathbb{Z}_{k}$. The forms $A$ and $C$ are both gauge fields, whose field strengths $d A$ and $d C$ vanish by the equations of motion. There are gauge transformations that shift the integrals of $A$ and $C$ over closed cycles by one. A gauge symmetry is just a redundancy in our description, but an invariant property of this theory is that it has line and 3-surface operators, given by $e^{i \oint A}$ and $e^{i \oint C}$, 
with $A$ and $C$ integrated over closed cycles. And, it has a 1 -form $\mathbb{Z}_{k}$ global symmetry that multiplies the line operators $e^{i \oint A}$ by $e^{2 \pi i / k}$, and a similar 3-form global symmetry.

When we put such a theory on $A d S_{5}$, we need to choose boundary conditions; the possible boundary conditions for such topological gauge theories were discussed in [23]. The variational principle implies that we need to set to zero $A \wedge C$ along the boundary. If we set to zero $C$ along the boundary, then the boundary value of $A$ gives a gauge field in the dual CFT, corresponding to a $\mathbb{Z}_{k}$ gauge symmetry in this CFT. With this boundary condition line operators in the bulk are allowed to approach the boundary and to become line operators in the CFT, while 3 -surface operators cannot approach the boundary, but can end on the boundary, giving 2-surface operators in the CFT. This is as expected for a $\mathbb{Z}_{k}$ gauge symmetry in four space-time dimensions.

On the other hand, if we set $A$ to zero on the boundary, we obtain a $\mathbb{Z}_{k}$ global symmetry in the dual CFT. The line operators ending on the boundary now give local operators in the $\mathrm{CFT}$, and the 1-form global symmetry in the bulk becomes a standard $\mathbb{Z}_{k}$ global symmetry in the CFT, under which these local operators are charged. The 3 -surface operators going to the boundary give 3 -surface operators in the CFT, which are domain walls between different vacua related by $\mathbb{Z}_{k}$.

Thus, whenever we have a $\mathbb{Z}_{k}$ gauge symmetry on $A d S_{5}$, there are two natural boundary conditions. One of them gives a conformal field theory with a $\mathbb{Z}_{k}$ global symmetry, and the other gives a conformal field theory with a $\mathbb{Z}_{k}$ gauge symmetry (and a $\mathbb{Z}_{k} 2$-form global symmetry). The second theory is related to the first one by gauging its $\mathbb{Z}_{k}$ global symmetry, and similarly the first one arises from the second by gauging its $\mathbb{Z}_{k} 2$-form global symmetry. When $k$ is not prime, there are also additional possible boundary conditions, corresponding to gauging subgroups of $\mathbb{Z}_{k}$.

In the context of our discussion in the previous section, this implies that the 'parent' theories and their $\mathbb{Z}_{k}$-gaugings should arise from the same holographic dual, just with different boundary conditions for the $\mathbb{Z}_{k}$ gauge fields. We will see in section 3.4 how the $\mathbb{Z}_{k}$ gauge fields of (3.1) arise in F-theory on $A d S_{5} \times\left(S^{5} \times T^{2}\right) / \mathbb{Z}_{k}$ from integrals of the type IIB five-form field $F_{5}$ on discrete cycles. Note that all this applies already to the $A d S_{5} \times S^{5} / \mathbb{Z}_{2}$ case discussed in [31]; typically in that case only the option of having a global $\mathbb{Z}_{2}$ symmetry, leading to the $\mathrm{SO}(2 N)$ gauge theory, is discussed.

Finally, note that a very similar story occurs already in type IIB string theory on $A d S_{5} \times S^{5}$, which includes a topological sector in the bulk corresponding to a 1-form $\mathbb{Z}_{N}$ gauge symmetry, with an action $\frac{i N}{2 \pi} B_{2} \wedge d C_{2}$ (where $B_{2}$ and $C_{2}$ are the 2-form fields of type IIB string theory) [32, 33]; this topological theory was discussed in section 6 of [23]. In this case both simple boundary conditions give rise to a 1 -form $\mathbb{Z}_{N}$ global symmetry in the dual $\mathrm{CFT}$, and the resulting theories are $\mathrm{SU}(N)$ and $\mathrm{SU}(N) / \mathbb{Z}_{N}$ gauge theories $[23,34,35]$. In this specific case there is also an option of coupling these theories to a continuous $\mathrm{U}(1)$ gauge symmetry, leading to a $\mathrm{U}(N)$ theory $[36,37]$. One can also quantize the topological theory in ways that do not lead to local field theories [38]. 


\subsection{Discrete torsion}

In [31], Witten showed how to characterize the variants of O3-planes by studying the discrete torsion on $S^{5} / \mathbb{Z}_{2}$. In this section we generalize this to $S^{5} / \mathbb{Z}_{k}$ S-folds, for $k=3,4,6$.

For $k=2$, the discrete torsion of the NSNS and of the RR three-form field strengths takes values in $H^{3}\left(S^{5} / \mathbb{Z}_{2}, \tilde{\mathbb{Z}}\right)$, where the tilde over $\mathbb{Z}$ means that the coefficient system is multiplied by $(-1)$ when we go around the $\mathbb{Z}_{2}$ torsion 3-cycle of $S^{5} / \mathbb{Z}_{2}$. For $k=3,4,6$, we have a $\mathbb{Z}_{k}$ torsion 3 -cycle in $S^{5} / \mathbb{Z}_{k}$, and we have an action of an element $\rho \in \operatorname{SL}(2, \mathbb{Z})$ on the NS-NS and R-R field strengths in $\mathbb{Z} \oplus \mathbb{Z}$ when we go around the $\mathbb{Z}_{k}$ torsion cycle of $S^{5} / \mathbb{Z}_{k}$. We can choose a specific form for this $\rho$, given, say, by $\rho=\left(\begin{array}{cc}-1 & 0 \\ 0 & -1\end{array}\right)$ for $k=2, \rho=\left(\begin{array}{cc}-1 & -1 \\ 1 & 0\end{array}\right)$ for $k=3, \rho=\left(\begin{array}{cc}0 & -1 \\ 1 & 0\end{array}\right)$ for $k=4$, and $\rho=\left(\begin{array}{cc}0 & -1 \\ 1 & 1\end{array}\right)$ for $k=6$. Note that the eigenvalues of these matrices are $\gamma$ and $\gamma^{-1}$. The discrete torsion is then given by $H^{3}\left(S^{5} / \mathbb{Z}_{k},(\mathbb{Z} \oplus \mathbb{Z})_{\rho}\right)$, and its computation is standard in mathematics. ${ }^{4}$

In general, $H^{*}\left(S^{2 n-1} / \mathbb{Z}_{k}, A\right)$ where $A$ is a $\mathbb{Z}_{k}$-module is given by the cohomology of the complex

$$
C^{0} \stackrel{1-t}{\longrightarrow} C^{1} \stackrel{1+t+\cdots+t^{k-1}}{\longrightarrow} C^{2} \stackrel{1-t}{\longrightarrow} \cdots \stackrel{1+t+\cdots+t^{k-1}}{\longrightarrow} C^{2 n-2} \stackrel{1-t}{\longrightarrow} C^{2 n-1}
$$

where all $C^{i} \simeq A, t$ is the generator of $\mathbb{Z}_{k}$, and the differential $d$ is alternately given by the multiplication by $1-t$ or by $1+t+\cdots+t^{k-1}$. It is easy to see that $d^{2}=$ $(1-t)\left(1+t+\cdots+t^{k-1}\right)=1-t^{k}=0$.

When $k=2, t$ is just the multiplication by -1 . Then $1+t=0$ and $1-t=2$, from which we conclude $H^{3}\left(S^{5} / \mathbb{Z}_{2},(\mathbb{Z} \oplus \mathbb{Z})_{\rho}\right)=\mathbb{Z}_{2} \oplus \mathbb{Z}_{2}$, reproducing four types of O3-planes.

When $k=3,4,6$, the action $\rho$ of the generator of $\mathbb{Z}_{k}$ on $\mathbb{Z} \oplus \mathbb{Z}$ obeys $1+\rho+\cdots+\rho^{k-1}=0$, and $\operatorname{det}(1-\rho)=3,2,1$ for $k=3,4,6$, respectively, and therefore

$$
H^{3}\left(S^{5} / \mathbb{Z}_{k},(\mathbb{Z} \oplus \mathbb{Z})_{\rho}\right)= \begin{cases}\mathbb{Z}_{3} & (k=3), \\ \mathbb{Z}_{2} & (k=4), \\ \mathbb{Z}_{1} & (k=6) .\end{cases}
$$

This gives the discrete torsion groups arising from the 3 -form fields of type IIB string theory on these S-folds.

For $k=3$, we have three different possibilities, but two non-trivial elements of $\mathbb{Z}_{3}$ are related by conjugation in $\operatorname{SL}(2, \mathbb{Z})$, so up to S-duality transformations there are just two types of S-folds with $k=3$ (in the same sense that up to S-duality there are just two types of O3-planes that give different theories for the D3-branes on them). For $k=4$, the cohomology is $\mathbb{Z}_{2}$, and therefore we expect two types of S-folds. Similarly, for $k=6$, there is only one type of S-fold.

\subsection{Generalized Pfaffians}

In the holographic duals of the S-folds, the discrete torsion described above corresponds to discrete 3 -form fluxes on the $S^{3} / \mathbb{Z}_{k}$ discrete 3-cycle in $S^{5} / \mathbb{Z}_{k}$, and it affects the spectrum

\footnotetext{
${ }^{4}$ The method to compute cohomologies with twisted coefficients is explained in e.g. Hatcher [39] chapter 3.H; the cell decomposition of generalized lens spaces, of which $S^{5} / \mathbb{Z}_{k}$ is one, is given in the same book, example 2.43. Section 5.2.1 of Davis-Kirk [40] was also quite helpful.
} 
of wrapped branes on this 3-cycle. We can use this to match the discussion of the previous subsection to our analysis of section 2 .

For $k=2$ this was analyzed in [31]. There is a $\mathbb{Z}_{2}$-torsion three-cycle of the form $S^{3} / \mathbb{Z}_{2}$ within $S^{5} / \mathbb{Z}_{2}$, and only when the discrete torsion is zero, i.e. when the 3 -plane is $O 3^{-}$, we can wrap a D3-brane on this cycle. By analyzing the properties of this wrapped D3-brane we find that it corresponds to a dimension $N$ operator in the dual CFT, which can be naturally identified with the Pfaffian operator, that only exists in the $\mathrm{SO}(2 N)$ theory but not in $\mathrm{SO}(2 N+1)$ or $\mathrm{Sp}(N)$ theories. Note that, according to the discussions above, this operator exists in the 'parent' $\mathrm{SO}(2 N)$ theory, but not after we gauge the $\mathbb{Z}_{2}$ to get the $\mathrm{O}(2 N)$ theory. So in the AdS dual it exists when we choose the boundary condition for the $\mathbb{Z}_{2}$ gauge theory in the bulk that gives a $\mathbb{Z}_{2}$ global symmetry, corresponding to the 'parent' theory, but not for the other boundary condition, that corresponds to the theory on the D3-branes.

The obstruction to wrap D3-branes on $S^{3} / \mathbb{Z}_{2}$ can be understood as follows. The NSNS 3 -form flux $G$ can in general be in a non-trivial cohomological class. But when pulled-back to the worldvolume of a single D3-brane, $G$ is the exterior derivative of a gauge-invariant object $B-F$, where $F$ is the gauge field on the D3-brane, and therefore the cohomology class $[G]$ should be trivial. The argument for the RR flux is the S-dual of this.

For all $k=3,4,6$, there is a $\mathbb{Z}_{k}$-torsion three-cycle in $S^{5} / \mathbb{Z}_{k}$ of the form $S^{3} / \mathbb{Z}_{k}$. When the discrete torsion is zero, there is no obstruction to wrapping a D3-brane on this cycle. The scaling dimension of this wrapped D3-brane can be easily found to be $k N / k=N$. This matches the scaling dimensions we found for the $\ell=1$ variants of section 2 . So we identify the S-fold with no discrete torsion with the $\ell=1$ case (either the 'parent' theory, or its $\mathbb{Z}_{k}$ gauging that gives the theory on the D3-branes, depending on the boundary conditions).

For $k=6$, there is nothing more to discuss, since $H^{3}\left(S^{5} / \mathbb{Z}_{k},(\mathbb{Z} \oplus \mathbb{Z})_{\rho}\right)$ itself is trivial, so we do not find any variant except $\ell=1$.

For $k=3$, when the discrete torsion in $H^{3}\left(S^{5} / \mathbb{Z}_{k},(\mathbb{Z} \oplus \mathbb{Z})_{\rho}\right)=\mathbb{Z}_{3}$ is non-trivial, we cannot wrap a D3-brane on this cycle. So this should correspond to the $\ell=3$ variant (with no discrete symmetries).

The most subtle is the $k=4$ case, when the discrete torsion is given by an element in $H^{3}\left(S^{5} / \mathbb{Z}_{k},(\mathbb{Z} \oplus \mathbb{Z})_{\rho}\right)=\mathbb{Z}_{2}$. We claim that the non-trivial element of this discrete torsion gives the $\ell=4$ variant of section 2 , not the $\ell=2$ variant.

To see this, it is instructive to recall why in the case $k=2$ we can wrap two D3-branes on $S^{3} / \mathbb{Z}_{2}$ even with the discrete torsion. Note that it is not just that for $N$ D3-branes wrapping on the same cycle, the triviality of $N \cdot[G]$ suffices. For one thing, if we have two D3-branes wrapping on the same locus with at least $\mathrm{U}(1) \times \mathrm{U}(1)$ unbroken, then $B-F_{1}$ and $B-F_{2}$ are both gauge-invariant (where $F_{1,2}$ are the $\mathrm{U}(1)$ gauge fields coming from the first and the second Chan-Paton factor), and therefore $[G]$ still needs to be zero.

To wrap two D3-branes on $S^{3} / \mathbb{Z}_{2}$ with discrete torsion consistently, one needs to require that the Chan-Paton indices 1,2 are interchanged when we go around the $\mathbb{Z}_{2}$ cycle. This means that in fact there is a single connected D3-brane of the shape $S^{3}$, which is wrapped on $S^{3} / \mathbb{Z}_{2}$ using a 2:1 quotient map. In this particular setting, we know how to judge the consistency of wrapping: on $S^{3},(B-F)$ is a gauge-invariant object, so $[G]$ should vanish 
there. What needs to be checked is then to pull back the spacetime $G$ using the map

$$
S^{3} \rightarrow S^{3} / \mathbb{Z}_{2} \rightarrow S^{5} / \mathbb{Z}_{2},
$$

where the first is the 2:1 projection and the second is the embedding. The result is zero, from the trivial fact that $H^{3}\left(S^{3}, \mathbb{Z}\right)=\mathbb{Z}$ does not have non-zero two-torsion elements. Thus we see that we can wrap two D3-branes in this way.

Now, let us come back to the $k=4$ case, and try to wrap two D3-branes on $S^{3} / \mathbb{Z}_{4}$ with discrete torsion, which should naively be possible since the discrete torsion lies in $\mathbb{Z}_{2}$. Again, if the Chan-Paton structure is trivial, we cannot wrap them. If we try to do the analogue of the $k=2$ case above, we can try wrapping two D3-branes such that the Chan-Paton indices 1 and 2 are exchanged. Just as above, this is equivalent to wrapping one D3-brane on $S^{3} / \mathbb{Z}_{2}$, covering $S^{3} / \mathbb{Z}_{4}$ with a 2:1 quotient map. To test the consistency of the wrapping, one needs to pull back the spacetime obstruction on $S^{5} / \mathbb{Z}_{4}$ via

$$
S^{3} / \mathbb{Z}_{2} \rightarrow S^{3} / \mathbb{Z}_{4} \rightarrow S^{5} / \mathbb{Z}_{4}
$$

One finds that the pull-back is non-trivial, showing that this embedding is inconsistent. ${ }^{5}$ It is clear that we can wrap four D3-branes on the discrete cycle, so this implies that the theory with this discrete torsion should be the $\ell=4$ variant discussed in section 2 , and that we cannot have $S$-folds with the $\ell=2$ variant.

This does not prove, we admit, that there are no other non-Abelian configurations on two D3-branes on $S^{3} / \mathbb{Z}_{4}$ that still allow the wrapping. But we will see that the choice $\ell=4$ for this discrete torsion variant matches with the M-theory computation below.

\subsection{Realization of the $\mathbb{Z}_{k}$ gauge theory in F-theory}

After these discussions we can understand how the $\mathbb{Z}_{k}$ gauge theory of section 3.1 arises in F-theory on $S^{5} / \mathbb{Z}_{k}$, when the discrete torsion in $H^{3}\left(S^{5} / \mathbb{Z}_{k},(\mathbb{Z} \oplus \mathbb{Z})_{\rho}\right)$ is zero. Recall that $H_{n}\left(S^{5} / \mathbb{Z}_{k}, \mathbb{Z}\right)$ is $\mathbb{Z}_{k}$ for $n=1$ and 3 , and its generator is $S^{1} / \mathbb{Z}_{k}$ and $S^{3} / \mathbb{Z}_{k}$, respectively.

We can wrap a D3-brane on these cycles to obtain a world 3-cycle and a worldline on $A d S_{5}$. (Note that the latter is the generalized Pfaffian particle discussed above.) Clearly, they both carry $\mathbb{Z}_{k}$ charges. We are then naturally led to identify the worldline as coupling to the 1-form $A$ and the world 3-cycle to the 3 -form $C$ in (3.1). For this identification to make sense, it should be the case that if we rotate the generalized Pfaffian around an $S^{1}$ that has a unit linking number with the world 3-cycle of the D3-brane wrapped on $S^{1} / \mathbb{Z}_{k}$, there should be a non-trivial $\mathbb{Z}_{k}$ holonomy of unit strength.

This can be seen as follows. A D3-brane wrapped on $S^{1} / \mathbb{Z}_{k}$ creates an $F_{5}$ flux given by the Poincaré dual of its worldvolume. In this case this is given by $s \otimes c$, where $s$ is the generator of $H^{1}\left(S^{1}, \mathbb{Z}\right)$ where this $S^{1}$ is linked in $A d S_{5}$ around the worldvolume of the D3-brane, and $c$ is the generator of $H^{4}\left(S^{5} / \mathbb{Z}_{k}, \mathbb{Z}\right)=\mathbb{Z}_{k}$.

Now, we use the cohomology long exact sequence associated to the short exact sequence $0 \rightarrow \mathbb{Z} \rightarrow \mathbb{R} \rightarrow \mathrm{U}(1) \rightarrow 0$ to conclude that there is a natural isomorphism

$$
H^{3}\left(S^{5} / \mathbb{Z}_{k}, \mathrm{U}(1)\right) \simeq H^{4}\left(S^{5} / \mathbb{Z}_{k}, \mathbb{Z}\right)=\mathbb{Z}_{k}
$$

\footnotetext{
${ }^{5}$ In general, the pull-back map from $S^{n} / \mathbb{Z}_{a b}$ to $S^{n} / \mathbb{Z}_{b}$ is a multiplication map by $1+t+t^{2}+\ldots+t^{a-1}$, where $t$ is the generator of the $\mathbb{Z}_{a}$ action that divides $S^{n} / \mathbb{Z}_{b}$ to $S^{n} / \mathbb{Z}_{a b}$.
} 
In physics terms, this means that the discrete $\mathbb{Z}_{k}$ field strength (which is $\mathbb{Z}$-valued) with four legs along $S^{5} / \mathbb{Z}_{k}$ can be naturally identified with the discrete $\mathbb{Z}_{k}$ holonomy (which is $\mathrm{U}(1)$-valued) with three legs along $S^{5} / \mathbb{Z}_{k}$.

From this we see that we have a holonomy of the type IIB $C_{4}$ field given by the element

$$
s \otimes c \in H^{1}\left(S^{1}, \mathbb{Z}\right) \otimes H^{3}\left(S^{5} / \mathbb{Z}_{k}, \mathrm{U}(1)\right),
$$

which can be naturally integrated on the cycle $S^{1} \times S^{3} / \mathbb{Z}_{k}$ to give $\exp (2 \pi i / k)$. This means that the generalized Pfaffian particle wrapped on $S^{3} / \mathbb{Z}_{k}$, when carried around the $S^{1}$ linking the worldvolume of the D3-brane wrapped on $S^{1} / \mathbb{Z}_{k}$, experiences this holonomy. This is indeed the behavior we expect for the objects charged under the $\mathbb{Z}_{k} 1$-form $A$ and the $\mathbb{Z}_{k}$ 3-form $C$ in (3.1).

\section{Comparison to M-theory}

In this section, we consider M-theory configurations on $\left(\mathbb{C}^{3} \times T^{2}\right) / \mathbb{Z}_{k}$. We know that we obtain such configurations from any of our S-folds on a circle, using the standard relation between type IIB theory on a circle and M-theory on a torus. However, the opposite is not true, since when we translate some configuration of discrete fluxes in M-theory on $\left(\mathbb{C}^{3} \times T^{2}\right) / \mathbb{Z}_{k}$ to F-theory on a circle, we could also have some non-trivial action of the shift around the F-theory circle, corresponding to a 'shift-S-fold' where there is a rotation on the transverse $\mathbb{C}^{3}$ when we go around the compactified $S^{1}$. By analyzing all possible discrete charges in M-theory and translating them to F-theory, we learn about all possible variants of S-folds.

\subsection{Discrete fluxes in M-theory on $\left(\mathbb{C}^{3} \times T^{2}\right) / \mathbb{Z}_{k}$}

Let us start by analyzing the possible discrete fluxes in M-theory, which come from 4 -form fluxes. For a given $k,\left(\mathbb{C}^{3} \times T^{2}\right) / \mathbb{Z}_{k}$ has several fixed points of the form $\mathbb{C}^{4} / \mathbb{Z}_{\ell_{i}}$, each of which has an associated $H^{4}\left(S^{7} / \mathbb{Z}_{\ell_{i}}, \mathbb{Z}\right)=\mathbb{Z}_{\ell_{i}}$ in M-theory [41]. From this viewpoint, the possible discrete charges are given by the orbifold actions on the fixed points,

$$
\bigoplus H^{3}\left(\mathbb{C}^{4} / \mathbb{Z}_{\ell_{i}}, \mathbb{Z}\right)= \begin{cases}\mathbb{Z}_{2} \oplus \mathbb{Z}_{2} \oplus \mathbb{Z}_{2} \oplus \mathbb{Z}_{2} & (k=2), \\ \mathbb{Z}_{3} \oplus \mathbb{Z}_{3} \oplus \mathbb{Z}_{3} & (k=3), \\ \mathbb{Z}_{4} \oplus \mathbb{Z}_{4} \oplus \mathbb{Z}_{2} & (k=4), \\ \mathbb{Z}_{6} \oplus \mathbb{Z}_{3} \oplus \mathbb{Z}_{2} & (k=6) .\end{cases}
$$

We can alternatively measure the same charges by considering $H^{4}$ of the 'asymptotic infinity' of $\left(\mathbb{C}^{3} \times T^{2}\right) / \mathbb{Z}_{k}$, which has the form $\left(S^{5} \times T^{2}\right) / \mathbb{Z}_{k}$. This is a $T^{2}$ bundle over $S^{5} / \mathbb{Z}_{k}$, and as such, one can apply the Leray-Serre spectral sequence, ${ }^{6}$ that says that it has the filtration

$$
H^{4}\left(\left(S^{5} \times T^{2}\right) / \mathbb{Z}_{k}, \mathbb{Z}\right)=F^{2,2} \supset F^{3,1} \supset F^{4,0},
$$

\footnotetext{
${ }^{6}$ For an introduction on the Leray-Serre spectral sequence, see e.g. [42]. In our case, the computation goes as follows. The second page $E_{2}^{p, q}$ of the spectral sequence is given by $E_{2}^{p, q}=H^{p}\left(S^{5} / \mathbb{Z}_{k}, H^{q}\left(T^{2}, \mathbb{Z}\right)\right)$, with the differentials $d_{2}^{p, q}: E_{2}^{p, q} \rightarrow E_{2}^{p+2, q-1}$. The third page is given by $E_{3}^{p, q}=\operatorname{Ker} d_{3}^{p, q} / \operatorname{Im} d^{p-2, q+1}$, and has the differentials $d_{3}^{p, q}: E_{3}^{p, q} \rightarrow E_{3}^{p+3, q-2}$. The second page and the part relevant for us of the third page
} 
where

$$
\begin{aligned}
F^{2,2} / F^{3,1} & =H^{2}\left(S^{5} / \mathbb{Z}_{k}, H^{2}\left(T^{2}, \mathbb{Z}\right)\right)=H^{2}\left(S^{5} / \mathbb{Z}_{k}, \mathbb{Z}\right), \\
F^{3,1} / F^{4,0} & =H^{3}\left(S^{5} / \mathbb{Z}_{k}, H^{1}\left(T^{2}, \mathbb{Z}\right)\right)=H^{3}\left(S^{5} / \mathbb{Z}_{k},(\mathbb{Z} \oplus \mathbb{Z})_{\rho}\right), \\
F^{4,0} & =H^{4}\left(S^{5} / \mathbb{Z}_{k}, H^{0}\left(T^{2}, \mathbb{Z}\right)\right)=H^{4}\left(S^{5} / \mathbb{Z}_{k}, \mathbb{Z}\right) .
\end{aligned}
$$

The different subgroups here correspond in a sense to the "number of legs along the base $S^{5} / \mathbb{Z}_{k}$ and the fiber $T^{2}$. As we are taking the $\mathbb{Z}$-valued cohomology, we only have a filtration and it is not guaranteed that the group (4.2) is a direct sum of (4.3)-(4.5).

We can easily compute (4.3)-(4.5) to obtain

$$
F^{2,2} / F^{3,1}=\mathbb{Z}_{k}, \quad F^{3,1} / F^{4,0}=\left\{\begin{array}{ll}
\mathbb{Z}_{2} \oplus \mathbb{Z}_{2} & (k=2) \\
\mathbb{Z}_{3} & (k=3) \\
\mathbb{Z}_{2} & (k=4) \\
\mathbb{Z}_{1} & (k=6)
\end{array} \quad F^{4,0}=\mathbb{Z}_{k} .\right.
$$

From the standard F-theory / M-theory mapping, we see that each piece (4.3)-(4.5) has the following effect in the F-theory language:

- The piece $F^{2,2} / F^{3,1}$ becomes a component of the metric of F-theory on $S^{1}$. Concretely, it specifies the amount of the rotation on the transverse space while we go around $S^{1}$. Therefore, the system is a plain $S^{1}$ compactification if the flux in this piece is zero, whereas it is a shift S-fold if it is non-zero.

- The piece $F^{3,1} / F^{4,0}$ becomes the discrete RR and NSNS 3-form fluxes around the Sfold. Indeed, the coefficient system $H^{1}\left(T^{2}, \mathbb{Z}\right)$ of the fiber is exactly the one $(\mathbb{Z} \oplus \mathbb{Z})_{\rho}$ that we discussed in the previous section, and there is a natural isomorphism of $F^{3,1} / F^{4,0}$ with the discrete torsion (3.3).

- The piece $F^{4,0}$ becomes an $F_{5}$ flux having one leg along the $S^{1}$. As discussed in section 3.4, we have a natural identification $H^{4}\left(S^{5} / \mathbb{Z}_{k}, \mathbb{Z}\right)=H^{3}\left(S^{5} / \mathbb{Z}_{k}, \mathrm{U}(1)\right)$. So, this piece can also be regarded as specifying a $C_{4}$ holonomy having one leg along the $S^{1}$.

Note that

$$
\left(F^{2,2} / F^{3,1}\right) \oplus\left(F^{3,1} / F^{4,0}\right) \oplus F^{4,0}= \begin{cases}\mathbb{Z}_{2} \oplus \mathbb{Z}_{2} \oplus \mathbb{Z}_{2} \oplus \mathbb{Z}_{2} & (k=2), \\ \mathbb{Z}_{3} \oplus \mathbb{Z}_{3} \oplus \mathbb{Z}_{3} & (k=3), \\ \mathbb{Z}_{4} \oplus \mathbb{Z}_{4} \oplus \mathbb{Z}_{2} & (k=4), \\ \mathbb{Z}_{6} \oplus \mathbb{Z}_{3} \oplus \mathbb{Z}_{2} & (k=6),\end{cases}
$$

are then given by

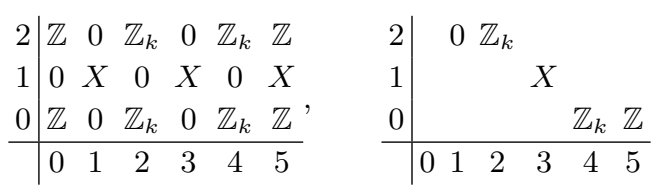

where $X=\mathbb{Z}_{2} \oplus \mathbb{Z}_{2}, \mathbb{Z}_{3}, \mathbb{Z}_{2}, \mathbb{Z}_{1}$ for $k=2,3,4,6$, respectively. From this we conclude that in fact $F^{p, q} / F^{p+1, q-1}=E_{\infty}^{p, q} \simeq E_{2}^{p, q}$ for $p+q=4$. 
and we see that (4.7) and (4.1) are the same as abstract groups. What remains is to figure out the precise mapping between the two. This is a purely geometrical question since it is just the relation of the $H^{4}$ of $\left(\mathbb{C}^{3} \times T^{2}\right) / \mathbb{Z}_{k}$ computed at the origin and at the asymptotic infinity. As we have not yet done this computation, we will use guesswork, and then verify that it leads to consistent results for the M2-brane and D3-brane charges of the various singularities.

The $k=2$ case can be worked out using the known properties of the orientifolds. In this case the map

$$
a: \bigoplus H^{3}\left(\mathbb{C}^{4} / \mathbb{Z}_{\ell_{i}}\right)=\left(\mathbb{Z}_{2}\right)^{4} \rightarrow F^{2,2} / F^{3,1}=\mathbb{Z}_{2}
$$

is given by the sum of the four $\mathbb{Z}_{2}$ 's, and the map

$$
b: F^{4,0}=\mathbb{Z}_{2} \rightarrow \bigoplus H^{3}\left(\mathbb{C}^{4} / \mathbb{Z}_{\ell_{i}}\right)=\left(\mathbb{Z}_{2}\right)^{4}
$$

is the diagonal embedding. Note that $a \circ b$ is indeed a zero map.

We assume that a similar relation holds also in the other cases. Namely, we assume that

$$
a: \bigoplus H^{3}\left(\mathbb{C}^{4} / \mathbb{Z}_{\ell_{i}}\right) \rightarrow F^{2,2} / F^{3,1}=\mathbb{Z}_{k}
$$

is the 'sum' and that

$$
b: F^{4,0}=\mathbb{Z}_{k} \rightarrow \bigoplus H^{3}\left(\mathbb{C}^{4} / \mathbb{Z}_{\ell_{i}}\right)
$$

is the 'diagonal embedding'. For $k=4$ and $k=6$, we need to use natural maps such as $\mathbb{Z}_{2} \rightarrow \mathbb{Z}_{6}$ and $\mathbb{Z}_{6} \rightarrow \mathbb{Z}_{2}$. We just choose a multiplication by 3 in the former, and the mod-2 map in the latter, and similarly for $k=4$. We see that the composition $a \circ b$ is indeed a zero map, which gives a small check of our assumptions.

\subsection{M2-brane and D3-brane charges}

Using the discussions above, let us test our identification by working out the M2-brane and D3-brane charges of our various configurations. We use the formula of [43] for the charge of $\mathbb{C}^{4} / \mathbb{Z}_{k}$ orbifolds with discrete torsion,

$$
(\text { M2-brane charge })=-\frac{1}{24}\left(k-\frac{1}{k}\right)+\frac{\ell(k-\ell)}{2 k} .
$$

The cases we use are

\begin{tabular}{|lr|cccccc|}
\hline & & $\ell=0$ & $\ell=1$ & $\ell=2$ & $\ell=3$ & $\ell=4$ & $\ell=5$ \\
\hline$k=2:$ & $-\frac{1}{16}+$ & 0 & $\frac{1}{4}$ & & & & \\
$k=3:$ & $-\frac{1}{9}+$ & 0 & $\frac{1}{3}$ & $\frac{1}{3}$ & & & \\
$k=4:$ & $-\frac{5}{32}+$ & 0 & $\frac{3}{8}$ & $\frac{1}{2}$ & $\frac{3}{8}$ & & \\
$k=6:$ & $-\frac{35}{144}+$ & 0 & $\frac{5}{12}$ & $\frac{2}{3}$ & $\frac{3}{4}$ & $\frac{2}{3}$ & $\frac{5}{12}$ \\
\hline
\end{tabular}

A long list of tables analyzing all possible cases follows. The end result is that every possible M-theory configuration can be interpreted as a plain $S^{1}$ compactification or a 
shift-S-fold on $S^{1}$ of precisely the S-folds that we discussed in the previous section (the types shaded in (2.13)), namely the S-folds with

$$
(k, \ell)=(2,1),(2,2),(3,1),(3,3),(4,1),(4,4), \text { and }(6,1) .
$$

In all cases we compute the D3-brane charge using the sum of the M2-brane charges of the orbifold singularities (4.12) and successfully compare it with our expectation (2.13).

\subsection{1 $k=2$}

Let us denote the discrete charges of the four fixed points as elements in $\left(\begin{array}{l}\mathbb{Z}_{2} \mathbb{Z}_{2} \\ \mathbb{Z}_{2}\end{array} \mathbb{Z}_{2}\right)$. The piece $F_{4,0}=\mathbb{Z}_{2}$ is generated by $\left(\begin{array}{ll}0 & 0 \\ 0 & 0\end{array}\right)$ and $\left(\begin{array}{ll}1 & 1 \\ 1 & 1\end{array}\right)$. The piece $F^{2,2} / F^{3,1}$ is given by the sum of the four entries. In the interpretations below, we consider the columns of the matrices to correspond to O2-planes, when we interpret our M-theory configuration in type IIA by shrinking one cycle of the torus (as we can do for $k=2$ ). Of course everything should work out correctly in this $k=2$ case, and has been already worked out in $[44,45]$. We reproduce the analysis here since it is a useful warm-up for $k=3,4,6$.

Shift $=0 / 2 \in F^{2,2} / F^{3,1}$ :

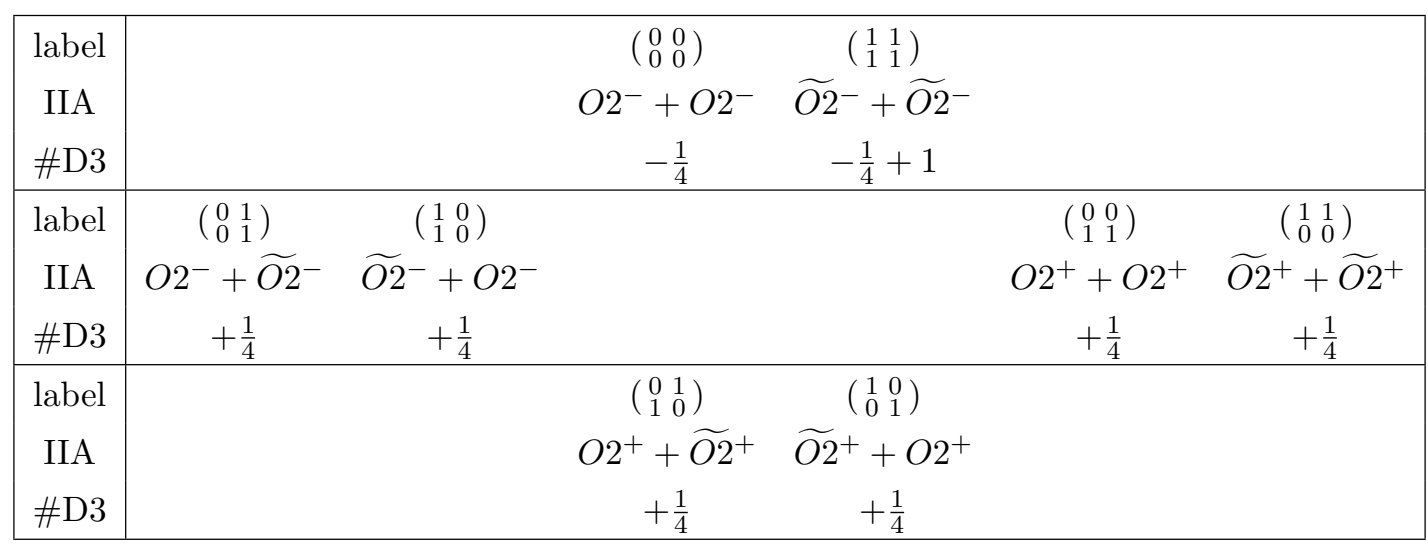

The first two come from $\mathrm{O}^{-}$, but the latter of the two has one additional mobile D3-brane stuck at the origin, due to a non-trivial Wilson line around $S^{1}$ in the component of $\mathrm{O}(2 N)$ disconnected from the identity. The rest all come from the other three O3-planes wrapped on $S^{1}$.

Shift $=1 / 2 \in F^{2,2} / F^{3,1}$ :

\begin{tabular}{|c|c|c|c|c|c|c|}
\hline $\begin{array}{l}\text { label } \\
\text { IIA } \\
\text { \#D3 }\end{array}$ & & & $\begin{array}{c}\left(\begin{array}{ll}1 & 0 \\
0 & 0\end{array}\right) \\
\widetilde{O 2^{+}}+O 2^{-} \\
0\end{array}$ & $\begin{array}{c}\left(\begin{array}{ll}0 & 1 \\
1 & 1\end{array}\right) \\
O 2^{-}+\widetilde{O 2^{-}} \\
0+\frac{1}{2}\end{array}$ & & \\
\hline label & $\left(\begin{array}{ll}1 & 1 \\
0 & 1\end{array}\right)$ & $\left(\begin{array}{ll}0 & 0 \\
1 & 0\end{array}\right)$ & & & $\left(\begin{array}{ll}1 & 0 \\
1 & 1\end{array}\right)$ & $\left(\begin{array}{ll}0 & 1 \\
0 & 0\end{array}\right)$ \\
\hline IIA & $\widetilde{O 2}^{+}+\widetilde{O 2^{-}}$ & $O 2^{+}+O 2^{-}$ & & & $\widetilde{O 2}^{-}+O 2^{+}$ & $O 2^{-}+\widetilde{O 2^{+}}$ \\
\hline \#D3 & $0+\frac{1}{2}$ & 0 & & & 0 & $0+\frac{1}{2}$ \\
\hline label & & & $\left(\begin{array}{ll}1 & 1 \\
1 & 0\end{array}\right)$ & $\left(\begin{array}{ll}0 & 0 \\
0 & 1\end{array}\right)$ & & \\
\hline IIA & & & $\widetilde{O 2}^{-}+\widetilde{O 2}^{+}$ & $O 2^{-}+O 2^{+}$ & & \\
\hline \#D3 & & & $0+\frac{1}{2}$ & 0 & & \\
\hline
\end{tabular}


They are $\mathbb{Z}_{2}$ shift-orientifolds around $S^{1}$. More precisely, the geometry is a $\mathbb{C}^{3}$ fibration over $S^{1}$ such that when we go around $S^{1}$, we have a multiplication by $(-1)$ on $\mathbb{C}^{3}$. The ones with D3-brane charge 0 are an empty shift-orientifold, and the ones with charge $0+1 / 2$ have one D3-brane wrapped around $S^{1}$. Note that the charge $1 / 2$ we are seeing here reflects the fact that the $T^{2}$ of M-theory is fibered over $\mathbb{C}^{3} / \mathbb{Z}_{2}$. In the type IIB frame, a fiber over a particular point on $S^{1}$ is $\mathbb{C}^{3}$, and therefore the D3-brane charge is 1 if we integrate over the asymptotic infinity of this $\mathbb{C}^{3}$.

\subsection{2 $k=3$}

Let us denote the discrete charges of the three fixed points as elements in $\left(\mathbb{Z}_{3}, \mathbb{Z}_{3}, \mathbb{Z}_{3}\right)$. The piece $F_{4,0}=\mathbb{Z}_{3}$ consists of $(0,0,0),(1,1,1),(2,2,2)=-(1,1,1)$. The piece $F^{2,2} / F^{3,1}=\mathbb{Z}_{3}$ corresponding to the shift is given by the sum of the entries. There are 27 choices in total.

Shift $=0 / 3 \in F^{2,2} / F^{3,1}$ :

\begin{tabular}{|c|ccc|}
\hline label & $(0,0,0)$ & $(1,1,1)$ & $(2,2,2)$ \\
\#D3 & $-\frac{1}{3}$ & $-\frac{1}{3}+1$ & $-\frac{1}{3}+1$ \\
\hline label & $(0,1,2)$ & $(1,2,0)$ & $(2,0,1)$ \\
\#D3 & $+\frac{1}{3}$ & $+\frac{1}{3}$ & $+\frac{1}{3}$ \\
\hline label & $(0,2,1)$ & $(1,0,2)$ & $(2,1,0)$ \\
\#D3 & $+\frac{1}{3}$ & $+\frac{1}{3}$ & $+\frac{1}{3}$ \\
\hline
\end{tabular}

Among the first three, the first entry has the right D3-brane charge to be the $(k=$ $3, \ell=1)$ S-fold, see (2.13). The other two have one more mobile D3-brane, stuck at the origin through a non-trivial $\mathbb{Z}_{3}$ background holonomy around $S^{1}$.

The others all have the right D3-charge to be the $(k=3, \ell=3)$ S-fold, see $(2.13)$ again. The second three and the third three have opposite $\mathbb{Z}_{3}$ charge characterizing the type of the S-fold.

Shift $=1 / 3 \in F^{2,2} / F^{3,1}$ :

\begin{tabular}{|c|ccc|}
\hline label & $(1,0,0)$ & $(2,1,1)$ & $(0,2,2)$ \\
\#D3 & 0 & $\frac{2}{3}$ & $\frac{1}{3}$ \\
\hline label & $(1,1,2)$ & $(2,2,0)$ & $(0,0,1)$ \\
\#D3 & $\frac{2}{3}$ & $\frac{1}{3}$ & 0 \\
\hline label & $(1,2,1)$ & $(2,0,2)$ & $(0,1,0)$ \\
\#D3 & $\frac{2}{3}$ & $\frac{1}{3}$ & 0 \\
\hline
\end{tabular}

They are the $\mathbb{Z}_{3}$ shift-S-folds, with the rotation angle $2 \pi / 3$, of the flat background on $S^{1}$, with 0, 1, 2 D3-brane(s) wrapped around at the origin. 
Shift $=2 / 3 \in F^{2,2} / F^{3,1}$ :

\begin{tabular}{|c|ccc|}
\hline label & $(2,0,0)$ & $(0,1,1)$ & $(1,2,2)$ \\
\#D3 & 0 & $\frac{2}{3}$ & $\frac{1}{3}$ \\
\hline label & $(2,1,2)$ & $(0,2,0)$ & $(1,0,1)$ \\
\#D3 & $\frac{2}{3}$ & 0 & $\frac{1}{3}$ \\
\hline label & $(2,2,1)$ & $(0,0,2)$ & $(1,1,0)$ \\
\#D3 & $\frac{2}{3}$ & 0 & $\frac{1}{3}$ \\
\hline
\end{tabular}

They are again the $\mathbb{Z}_{3}$ shift-S-folds, but with the rotation angle $4 \pi / 3$, of the flat background on $S^{1}$, with $0,1,2$ mobile D3-brane(s) wrapped around at the origin.

\subsection{3 $k=4$}

We denote the charges at the fixed points as elements in $\left(\mathbb{Z}_{4}, \mathbb{Z}_{4} ; \mathbb{Z}_{2}\right)$. The piece $F_{4,0}=\mathbb{Z}_{4}$ consists of $(0,0 ; 0),(1,1 ; 1),(2,2 ; 0),(3,3 ; 1)$.

Shift $=0 / 4 \in F^{2,2} / F^{3,1}$ :

\begin{tabular}{|c|cccc|}
\hline label & $(0,0 ; 0)$ & $(1,1 ; 1)$ & $(2,2 ; 0)$ & $(3,3 ; 1)$ \\
\#D3 & $-\frac{3}{8}$ & $-\frac{3}{8}+1$ & $-\frac{3}{8}+1$ & $-\frac{3}{8}+1$ \\
\hline label & $(0,2 ; 1)$ & $(1,3 ; 0)$ & $(2,0 ; 1)$ & $(3,1 ; 0)$ \\
\#D3 & $+\frac{3}{8}$ & $+\frac{3}{8}$ & $+\frac{3}{8}$ & $+\frac{3}{8}$ \\
\hline
\end{tabular}

Among the first four, the first has the right D3-brane charge to be the $(k=4, \ell=1)$ S-fold, see (2.13). The other three have one more mobile D3-brane, stuck at the origin through non-trivial $\mathbb{Z}_{4}$ background holonomy around $S^{1}$. All the second four have the right D3-charge to be the $(k=4, \ell=4)$ S-fold, see (2.13).

Shift $=1 / 4 \in F^{2,2} / F^{3,1}$ :

\begin{tabular}{|c|cccc|}
\hline label & $(0,1 ; 0)$ & $(1,2 ; 1)$ & $(2,3 ; 0)$ & $(3,0 ; 1)$ \\
\#D3 & 0 & $+\frac{3}{4}$ & $+\frac{1}{2}$ & $+\frac{1}{4}$ \\
\hline label & $(0,3 ; 1)$ & $(1,0 ; 0)$ & $(2,1 ; 1)$ & $(3,2 ; 0)$ \\
\#D3 & $+\frac{1}{4}$ & 0 & $+\frac{3}{4}$ & $+\frac{1}{2}$ \\
\hline
\end{tabular}

These are $\mathbb{Z}_{4}$ shift-S-folds of flat space, with rotation angle $\pi / 2$, and with $0,1,2$ or 3 mobile D3-brane(s) around $S^{1}$.

Shift $=2 / 4 \in F^{2,2} / F^{3,1}$ :

\begin{tabular}{|c|cccc|}
\hline label & $(0,0 ; 1)$ & $(1,1 ; 0)$ & $(2,2 ; 1)$ & $(3,3 ; 0)$ \\
\#D3 & $-\frac{1}{8}$ & $-\frac{1}{8}+\frac{1}{2}$ & $-\frac{1}{8}+1$ & $-\frac{1}{8}+\frac{1}{2}$ \\
\hline label & $(0,2 ; 0)$ & $(1,3 ; 1)$ & $(2,0 ; 0)$ & $(3,1 ; 1)$ \\
\#D3 & $+\frac{1}{8}$ & $+\frac{1}{8}+\frac{1}{2}$ & $+\frac{1}{8}$ & $+\frac{1}{8}+\frac{1}{2}$ \\
\hline
\end{tabular}

These are the $\mathbb{Z}_{2}$ shift-S-folds on $S^{1}$ of the O3-planes. 
Among the first four, the first has the right D3-brane charge to be the $\mathbb{Z}_{2}$ shift-Sfold of $\mathrm{O3}^{-}$. Recall that the base of the M-theory configuration is $\mathbb{C}^{3} / \mathbb{Z}_{4}$, but the in the type IIB description, the fiber at a particular point on $S^{1}$ is $\mathbb{C}^{3} / \mathbb{Z}_{2}$. The D3-brane charge of $\mathrm{O3}^{-}$is $-1 / 4$, and therefore the charge as seen from the M-theory configuration is $1 / 2 \cdot(-1 / 4)=-1 / 8$.

The others have one or two additional mobile D3-brane(s) trapped at the origin. Among the second four, the first and the third have the right D3-brane charge to be the $\mathbb{Z}_{2}$ shift-S-fold of $\widetilde{\mathrm{O3}^{+}}$. The second and the fourth have one additional mobile D3-brane trapped at the origin.

Note that we cannot take the $\mathbb{Z}_{2}$ shift S-fold of $\widetilde{\mathrm{O3}^{-}}$or $\mathrm{O3}^{+}$, since they are exchanged by this $\mathbb{Z}_{2}$ operation.

Shift $=3 / 4 \in F^{2,2} / F^{3,1}$ :

\begin{tabular}{|c|cccc|}
\hline label & $(0,1 ; 1)$ & $(1,2 ; 0)$ & $(2,3 ; 1)$ & $(3,0 ; 0)$ \\
\#D3 & $+\frac{1}{4}$ & $+\frac{1}{2}$ & $+\frac{3}{4}$ & 0 \\
\hline label & $(0,3 ; 0)$ & $(1,0 ; 1)$ & $(2,1 ; 0)$ & $(3,2 ; 1)$ \\
\#D3 & 0 & $+\frac{1}{4}$ & $+\frac{1}{2}$ & $+\frac{3}{4}$ \\
\hline
\end{tabular}

These are $\mathbb{Z}_{4}$ shift-S-folds of flat space, with rotation angle $3 \pi / 2$, and with $0,1,2$ or 3 mobile D3-brane(s) around $S^{1}$.

\subsection{4 $k=6$}

We denote the charges at the fixed points as elements in $\left(\mathbb{Z}_{6}, \mathbb{Z}_{3}, \mathbb{Z}_{2}\right)$. The piece $F_{4,0}=\mathbb{Z}_{6}$ consists of $(0,0,0),(1,1,1),(2,2,0),(3,0,1),(4,1,0),(5,2,1)$.

Shift $=0 / 6 \in F^{2,2} / F^{3,1}$ :

$$
\begin{array}{|c|cccccc|}
\hline \text { label } & (0,0,0) & (1,1,1) & (2,2,0) & (3,0,1) & (4,1,0) & (5,2,1) \\
\text { \#D3 } & -\frac{5}{12} & -\frac{5}{12}+1 & -\frac{5}{12}+1 & -\frac{5}{12}+1 & -\frac{5}{12}+1 & -\frac{5}{12}+1 \\
\hline
\end{array}
$$

The first has the right D3-brane charge to be the $(k=6, \ell=1)$ S-fold, see (2.13). The other five have one more mobile D3-brane, stuck at the origin through a non-trivial $\mathbb{Z}_{6}$ background holonomy around $S^{1}$.

Shift $=1 / 6 \in F^{2,2} / F^{3,1}$ :

\begin{tabular}{|c|cccccc|}
\hline label & $(1,0,0)$ & $(2,1,1)$ & $(3,2,0)$ & $(4,0,1)$ & $(5,1,0)$ & $(0,2,1)$ \\
\#D3 & 0 & $\frac{5}{6}$ & $\frac{2}{3}$ & $\frac{1}{2}$ & $\frac{1}{3}$ & $\frac{1}{6}$ \\
\hline
\end{tabular}

This is the $\mathbb{Z}_{6}$ shift-S-fold of flat space, with zero to five D3-branes stuck at the origin. The rotation angle is $2 \pi / 6$. 
Shift $=2 / 6 \in F^{2,2} / F^{3,1}$ :

\begin{tabular}{|c|cccccc|}
\hline label & $(2,0,0)$ & $(3,1,1)$ & $(4,2,0)$ & $(5,0,1)$ & $(0,1,0)$ & $(1,2,1)$ \\
\#D3 & $-\frac{1}{12}+\frac{1}{3}$ & $-\frac{1}{12}+1$ & $-\frac{1}{12}+\frac{2}{3}$ & $-\frac{1}{12}+\frac{1}{3}$ & $-\frac{1}{12}$ & $-\frac{1}{12}+\frac{2}{3}$ \\
\hline
\end{tabular}

The fifth has the correct charge to be the $\mathbb{Z}_{3}$ shift-S-fold of the standard $03^{-}$-plane. Since the $O 3^{-}$-plane itself has an identification by the angle $\pi$, its $\mathbb{Z}_{3}$ quotient involves the rotation by $\pi / 3$ of the transverse space. Note also that the $O 3^{-}$-plane has the D3-brane charge $-1 / 4$, therefore we see $1 / 3 \cdot(-1 / 4)=-1 / 12$ in M-theory. The others have one or two additional D3-brane(s) at the origin.

Shift $=3 / 6 \in F^{2,2} / F^{3,1}$ :

\begin{tabular}{|c|cccccc|}
\hline label & $(3,0,0)$ & $(4,1,1)$ & $(5,2,0)$ & $(0,0,1)$ & $(1,1,0)$ & $(2,2,1)$ \\
\#D3 & $-\frac{1}{6}+\frac{1}{2}$ & $-\frac{1}{6}+1$ & $-\frac{1}{6}+\frac{1}{2}$ & $-\frac{1}{6}$ & $-\frac{1}{6}+\frac{1}{2}$ & $-\frac{1}{6}+1$ \\
\hline
\end{tabular}

The fourth has the correct charge to be the $\mathbb{Z}_{2}$ shift-S-fold of the $(k=3, \ell=1)$ S-fold. The others have one or two additional mobile D3-brane(s) on top.

Shift $=4 / 6 \in F^{2,2} / F^{3,1}$ :

\begin{tabular}{|c|cccccc|}
\hline label & $(4,0,0)$ & $(5,1,1)$ & $(0,2,0)$ & $(1,0,1)$ & $(2,1,0)$ & $(3,2,1)$ \\
\#D3 & $-\frac{1}{12}+\frac{1}{3}$ & $-\frac{1}{12}+\frac{2}{3}$ & $-\frac{1}{12}$ & $-\frac{1}{12}+\frac{1}{3}$ & $-\frac{1}{12}+\frac{2}{3}$ & $-\frac{1}{12}+1$ \\
\hline
\end{tabular}

The third has the correct charge to be the $\mathbb{Z}_{3}$ shift-S-fold, of rotation angle $2 \pi / 3$, of the standard $\mathrm{O3}^{-}$-plane. The others have one or two additional D3-brane(s) at the origin.

Shift $=5 / 6 \in F^{2,2} / F^{3,1}$ :

\begin{tabular}{|c|cccccc|}
\hline label & $(5,0,0)$ & $(0,1,1)$ & $(1,2,0)$ & $(2,0,1)$ & $(3,1,0)$ & $(4,2,1)$ \\
\#D3 & 0 & $\frac{1}{6}$ & $\frac{1}{3}$ & $\frac{1}{2}$ & $\frac{2}{3}$ & $\frac{5}{6}$ \\
\hline
\end{tabular}

This is the $\mathbb{Z}_{6}$ shift-S-fold of flat space, with zero to five D3-branes stuck at the origin. The rotation angle is $2 \pi \cdot 5 / 6$.

Comments: note that in the cases with shift $2,4 \in \mathbb{Z}_{6}$, we only find $\mathbb{Z}_{3}$ shift-S-folds of $O 3^{-}$, but we do not have $\mathbb{Z}_{3}$ shift-S-folds of $\widetilde{O 3}-\mathrm{O3}^{+}$and $\widetilde{O 3^{+}}$. This is as it should be, because these three types of O3-planes are permuted by the $\mathbb{Z}_{3}$ action.

Similarly, there is no $\mathbb{Z}_{2}$ shift-orientifold of the $(k=3, \ell=3)$ orientifold, since the $\mathbb{Z}_{2}$ action exchanges the two subtly different versions that we denoted by $(1,1,1)$ and $(2,2,2)$ in section 4.2.2. 


\section{Special cases and $\mathcal{N}=4$ enhancement}

So far, we saw that $N$ D3-branes probing various variants of the $\mathrm{S}$-folds give rise to $\mathcal{N}=3$ superconformal field theories characterized by

$$
(k, \ell)=(3,1),(3,3),(4,1),(4,4),(6,1) .
$$

When $\ell<k$ we expect to have both 'parent' theories and their discrete gaugings. In this section we discuss some interesting special cases, including cases where the $\mathcal{N}=3$ supersymmetry is enhanced to $\mathcal{N}=4$.

As discussed in [5], an enhancement of supersymmetry to $\mathcal{N}=4$ occurs if and only if there is a Coulomb branch operator of dimension 1 or 2 , since $\mathcal{N}=3$ supersymmetry then dictates the presence of extra supercharges. The dimensions of the Coulomb branch operators of our 'parent' $\mathcal{N}=3$ theories were given in (2.11). For $k=2$ we always have such an enhancement, but for $k=3,4,6$ we see that it happens just for $\ell=1$ and $N=1,2$. Note that the theory with the lowest central charge which does not have any enhancement is the $N=1, k=\ell=3$ theory, whose only Coulomb branch operator has dimension 3 . The central charges of this theory are the same as those of five vector multiplets. Since the Coulomb branch operators of $\mathcal{N}=3$ theories must be integers as shown in section 3.1 of [28], then this must be the 'minimal' $\mathcal{N}=3$ SCFT, assuming the general validity of the formula (2.3). It would be interesting to test this by a superconformal bootstrap analysis, generalizing the ones in $[46,47]$.

Going back to theories with $\mathcal{N}=4$ supersymmetry, the case $N=1$ is rather trivial: we just have a Coulomb branch operator of dimension one, so the moduli space is just $\mathbb{C}^{3}$, and we get the $\mathcal{N}=4$ super Yang-Mills theory with gauge group U(1). So let us discuss the $N=2$ cases.

The spectrum of the Coulomb branch operators of the 'parent' theory is given by

$$
\begin{cases}2,3 & (k=3), \\ 2,4 & (k=4), \\ 2,6 & (k=6) .\end{cases}
$$

These spectra agree with those of an $\mathcal{N}=4$ super Yang-Mills theory with gauge group $\mathrm{SU}(3), \mathrm{SO}(5)$ and $G_{2}$, respectively. Below we give evidence that indeed, the 'parents' of these S-fold configurations give rise to these $\mathcal{N}=4$ super Yang-Mills theories, realized in a somewhat unusual manner. Note that $\mathcal{N}=4$ theories always have an exactly marginal deformation, sitting in the same multiplet as the dimension two Coulomb branch operator, and our conjectured relation implies that for our $N=2$ theories this is the gauge coupling of these $\mathcal{N}=4$ gauge theories. Our discussion in the previous sections implies that in the AdS dual of these 'parent' theories, this marginal deformation corresponds to a scalar field coming from a D3-brane wrapped on the torsion 3-cycle; for this specific case this wrapped D3-brane gives rise to a massless field.

Again let us limit ourselves to the points on the moduli space corresponding to a Coulomb branch from the point of view of an $\mathcal{N}=2$ description of our SCFTs. In 
the $N=2, \ell=1$ theories, this subspace is parameterized by $z_{1,2} \in \mathbb{C}$, with the gauge symmetry $(2.10)$

$$
\left(z_{1}, z_{2}\right) \mapsto\left(\gamma^{n} z_{2}, \gamma^{-n} z_{1}\right)
$$

where $\gamma=e^{2 \pi i / k}$ and $n$ is any integer. The charges in a basis that is natural from this point of view can be written as $\left(e_{1}, m_{1} ; e_{2}, m_{2}\right)$.

Naively, one would expect the electric charges $e_{1}, e_{2}$ to correspond to electric charges of the corresponding $\mathcal{N}=4$ theory, but this cannot be the case because of the non-trivial $\mathrm{Sp}(4, \mathbb{Z})$ action on these charges, induced by the $\mathrm{SL}(2, \mathbb{Z})$ transformation that accompanies the identification (5.3). So instead we consider the rank-2 sublattice containing charges of the form $(Q ; \bar{Q})$ where we regard $(e, m) \in \mathbb{Z} \oplus \mathbb{Z}$ as a complex number $Q=e+m \gamma$.

Two charges from this sublattice are local with respect to each other. To see this, note that given $Q=e+m \gamma$ and $Q^{\prime}=e^{\prime}+m^{\prime} \gamma$, their Dirac pairing is

$$
e m^{\prime}-e^{\prime} m=\left(\bar{Q} Q^{\prime}-\bar{Q}^{\prime} Q\right) /(\gamma-\bar{\gamma})
$$

Then the Dirac pairing between the two charges $(Q ; \bar{Q})$ and $\left(Q^{\prime} ; \bar{Q}^{\prime}\right)$ is clearly zero. This means that we can take these charges to be the "electric charges" in the $\mathcal{N}=4$ description. We provide some consistency checks for this below.

Now, note that the $\operatorname{SL}(2, \mathbb{Z})$ element associated to the $\mathbb{Z}_{k}$ orbifold then acts on $Q$ just by multiplication by $\gamma$. Then the gauge transformation (5.3) acts on this variable $Q$ as

$$
Q \mapsto \gamma^{n} \bar{Q},
$$

which is a reflection of the complex plane along the line $e^{\pi i n / k} \mathbb{R}$. This makes it clear that the group generated by (5.3) for $k=3,4,6$ is the Weyl group of $\mathrm{SU}(3), \mathrm{SO}(5)$ and $G_{2}$, respectively.

Let us test our identification by looking at half-BPS particles. There's no string connecting $z_{1}$ and $\gamma^{n} z_{1}$, since we know nothing happens when $z_{1}=0$ for $\ell=1$. So there are only strings connecting $\gamma^{n} z_{1}$ and $\gamma^{m} z_{2}$. Using (5.3) we can always restrict $z_{1}$ to have a phase between 0 and $2 \pi / k$. Then we just have to consider all $(p, q)$-strings connecting $z_{1}$ and $\gamma^{n} z_{2}$ for $n=0, \ldots, k-1$. Since the IIB coupling constant is $\tau=\gamma$, the central charges of half-BPS particles are given by

$$
(p+q \gamma)\left(z_{1}-\gamma^{n} z_{2}\right)
$$

We conjectured that "electric" states have the charge $(Q ; \bar{Q})$, and then their central charges are given by $Q z_{1}-\bar{Q} z_{2}$. Comparing with (5.6), we see that "electric" objects have the following $Q$ :

- For $k=3, Q=\omega^{n}$ and $Q=(1+\omega) \omega^{n}=-\omega^{n-1}$ where $\omega=e^{2 \pi i / 3}$,

- For $k=4, Q=i^{n}$ and $Q=(1+i) i^{n}$,

- For $k=6, Q=\gamma^{n}$ and $Q=(1+\gamma) \gamma^{n}$.

Clearly they can be identified with the roots of $\mathrm{SU}(3), \mathrm{SO}(5)$, and $G_{2}$, respectively. 
The metric on the moduli space is also correctly mapped to that on the Cartan subalgebra of these groups. The original metric is $d z_{1} d \bar{z}_{1}+d z_{2} d \bar{z}_{2}$ on $\mathbb{C}^{2}$, and we choose a real subspace $\mathbb{R}^{2}$ of the form $Q z_{1}-\bar{Q} z_{2}$. Then, two vectors $Q z_{1}-\bar{Q} z_{2}$ and $Q^{\prime} z_{1}-\bar{Q}^{\prime} z_{2}$ in $\mathbb{R}^{2}$ have the induced inner product $\left(2 \operatorname{Re}\left(Q \bar{Q}^{\prime}\right)\right)$. Using this, we can easily check that the vectors listed above have the same inner products as the root vectors of $\mathrm{SU}(3), \mathrm{SO}(5)$, and $G_{2}$, in the normalization that the short roots have length squared 2 .

Finally, recall that the dyons of $\mathcal{N}=4 \mathrm{SYM}$ have central charges of the form

$$
\left(p+q \tau_{Y M}\right)\left(\alpha_{s} \cdot \phi\right), \quad\left(p+q \frac{\tau_{Y M}}{r}\right)\left(\alpha_{l} \cdot \phi\right),
$$

where $\alpha_{s, l}$ are short and long roots, and $r$ is the length squared of the long roots divided by that of the short roots. We can check that the spectrum (5.6) can be matched with (5.7) with the identification of the roots given above, if we take $\tau_{Y M}=-1 /(1+\gamma)$, uniformly for $k=3,4,6$. As a further check, note that for $k=6$ this is exactly the value of $\tau_{Y M}$ for which the $G_{2} \mathcal{N}=4$ theory has a discrete $\mathbb{Z}_{6}$ symmetry [48].

\section{Acknowledgments}

The authors would like to thank I. García-Etxebarria, D. Harlow, Z. Komargodski, T. Nishinaka, H. Ooguri and N. Seiberg for useful discussions. The work of OA is supported in part by an Israel Science Foundation center for excellence grant, by the I-CORE program of the Planning and Budgeting Committee and the Israel Science Foundation (grant number 1937/12), by the Minerva foundation with funding from the Federal German Ministry for Education and Research, by a Henri Gutwirth award from the Henri Gutwirth Fund for the Promotion of Research, and by the ISF within the ISF-UGC joint research program framework (grant no. 1200/14). OA is the Samuel Sebba Professorial Chair of Pure and Applied Physics. The work of YT is partially supported in part by JSPS Grant-in-Aid for Scientific Research No. 25870159, and by WPI Initiative, MEXT, Japan at IPMU, the University of Tokyo.

Open Access. This article is distributed under the terms of the Creative Commons Attribution License (CC-BY 4.0), which permits any use, distribution and reproduction in any medium, provided the original author(s) and source are credited.

\section{References}

[1] P. Argyres, M. Lotito, Y. Lü and M. Martone, Geometric constraints on the space of $N=2$ SCFTs I: physical constraints on relevant deformations, arXiv:1505.04814 [INSPIRE].

[2] P.C. Argyres, M. Lotito, Y. Lü and M. Martone, Geometric constraints on the space of $N=2$ SCFTs II: Construction of special Kähler geometries and $R G$ flows, arXiv: 1601.00011 [INSPIRE].

[3] P.C. Argyres, M. Lotito, Y. Lü and M. Martone, Expanding the landscape of $\mathcal{N}=2$ rank 1 SCFTs, JHEP 05 (2016) 088 [arXiv: 1602.02764] [INSPIRE].

[4] D. Gaiotto, $N=2$ dualities, JHEP 08 (2012) 034 [arXiv:0904.2715] [INSPIRE]. 
[5] O. Aharony and M. Evtikhiev, On four dimensional $N=3$ superconformal theories, JHEP 04 (2016) 040 [arXiv: 1512.03524] [INSPIRE].

[6] C. Cordova, T.T. Dumitrescu and K. Intriligator, Deformations of superconformal theories, arXiv: 1602.01217 [INSPIRE].

[7] I. García-Etxebarria and D. Regalado, $\mathcal{N}=3$ four dimensional field theories, JHEP 03 (2016) 083 [arXiv: 1512.06434] [INSPIRE].

[8] C.M. Hull, A geometry for non-geometric string backgrounds, JHEP 10 (2005) 065 [hep-th/0406102] [INSPIRE].

[9] A. Dabholkar and C. Hull, Duality twists, orbifolds and fluxes, JHEP 09 (2003) 054 [hep-th/0210209] [INSPIRE].

[10] C.M. Hull and A. Catal-Ozer, Compactifications with S duality twists, JHEP 10 (2003) 034 [hep-th/0308133] [INSPIRE].

[11] C.M. Hull and R.A. Reid-Edwards, Flux compactifications of M-theory on twisted tori, JHEP 10 (2006) 086 [hep-th/0603094] [INSPIRE].

[12] A. Catal-Ozer, Duality twists on a group manifold, JHEP 10 (2006) 072 [hep-th/0606278] [INSPIRE].

[13] R.A. Reid-Edwards, Geometric and non-geometric compactifications of IIB supergravity, JHEP 12 (2008) 043 [hep-th/0610263] [INSPIRE].

[14] O.J. Ganor and Y.P. Hong, Selfduality and Chern-Simons theory, arXiv:0812.1213 [INSPIRE].

[15] O.J. Ganor, Y.P. Hong and H.S. Tan, Ground states of S-duality twisted $N=4$ super Yang-Mills theory, JHEP 03 (2011) 099 [arXiv: 1007.3749] [INSPIRE].

[16] O.J. Ganor, Y.P. Hong, R. Markov and H.S. Tan, Static charges in the low-energy theory of the S-duality twist, JHEP 04 (2012) 041 [arXiv:1201.2679] [INSPIRE].

[17] C. Vafa, Evidence for F-theory, Nucl. Phys. B 469 (1996) 403 [hep-th/9602022] [InSPIRE].

[18] A. Fayyazuddin and M. Spalinski, Large- $N$ superconformal gauge theories and supergravity orientifolds, Nucl. Phys. B 535 (1998) 219 [hep-th/9805096] [INSPIRE].

[19] O. Aharony, A. Fayyazuddin and J.M. Maldacena, The large- $N$ limit of $N=2, N=1$ field theories from three-branes in F-theory, JHEP 07 (1998) 013 [hep-th/9806159] [INSPIRE].

[20] O. Aharony and Y. Tachikawa, A holographic computation of the central charges of $D=4$, $N=2$ SCFTs, JHEP 01 (2008) 037 [arXiv:0711.4532] [INSPIRE].

[21] P.C. Argyres and J.R. Wittig, Infinite coupling duals of $N=2$ gauge theories and new rank 1 superconformal field theories, JHEP 01 (2008) 074 [arXiv:0712.2028] [INSPIRE].

[22] A.D. Shapere and Y. Tachikawa, Central charges of $N=2$ superconformal field theories in four dimensions, JHEP 09 (2008) 109 [arXiv:0804.1957] [INSPIRE].

[23] D. Gaiotto, A. Kapustin, N. Seiberg and B. Willett, Generalized global symmetries, JHEP 02 (2015) 172 [arXiv: 1412.5148] [INSPIRE].

[24] T. Banks and N. Seiberg, Symmetries and strings in field theory and gravity, Phys. Rev. D 83 (2011) 084019 [arXiv: 1011.5120] [INSPIRE].

[25] S. Cecotti and M. Del Zotto, Higher S-dualities and Shephard-Todd groups, JHEP 09 (2015) 035 [arXiv: 1507.01799] [INSPIRE].

[26] G.I. Lehrer and D.E. Taylor, Unitary reflection groups, Australian Mathematical Society Lecture Series volume 20, Cambridge University Press, Cambridge U.K. (2009).

[27] C. Beem et al., Infinite chiral symmetry in four dimensions, Commun. Math. Phys. 336 (2015) 1359 [arXiv: 1312.5344] [INSPIRE]. 
[28] T. Nishinaka and Y. Tachikawa, On $4 D$ rank-one $N=3$ superconformal field theories, arXiv: 1602.01503 [INSPIRE].

[29] E. Witten, $\mathrm{SL}(2, \mathbb{Z})$ action on three-dimensional conformal field theories with Abelian symmetry, hep-th/0307041 [INSPIRE].

[30] D. Harlow and H. Ooguri, work in progress.

[31] E. Witten, Baryons and branes in Anti-de Sitter space, JHEP 07 (1998) 006 [hep-th/9805112] [INSPIRE].

[32] D.J. Gross and H. Ooguri, Aspects of large- $N$ gauge theory dynamics as seen by string theory, Phys. Rev. D 58 (1998) 106002 [hep-th/9805129] [inSPIRE].

[33] O. Aharony and E. Witten, Anti-de Sitter space and the center of the gauge group, JHEP 11 (1998) 018 [hep-th/9807205] [INSPIRE].

[34] O. Aharony, N. Seiberg and Y. Tachikawa, Reading between the lines of four-dimensional gauge theories, JHEP 08 (2013) 115 [arXiv:1305.0318] [INSPIRE].

[35] A. Kapustin and N. Seiberg, Coupling a QFT to a TQFT and duality, JHEP 04 (2014) 001 [arXiv: 1401.0740] [INSPIRE].

[36] J.M. Maldacena, G.W. Moore and N. Seiberg, D-brane charges in five-brane backgrounds, JHEP 10 (2001) 005 [hep-th/0108152] [INSPIRE].

[37] D. Belov and G.W. Moore, Conformal blocks for AdS $S_{5}$ singletons, hep-th/0412167 [INSPIRE].

[38] E. Witten, AdS/CFT correspondence and topological field theory, JHEP 12 (1998) 012 [hep-th/9812012] [INSPIRE].

[39] A. Hatcher, Algebraic topology, Cambridge University Press, Cambridge U.K. (2002).

[40] J.F. Davis and P. Kirk, Lecture notes in algebraic topology, Graduate Studies in Mathematics volume 35, American Mathematical Society, U.S.A. (2001).

[41] O. Aharony, O. Bergman and D.L. Jafferis, Fractional M2-branes, JHEP 11 (2008) 043 [arXiv: 0807.4924] [INSPIRE].

[42] A. Hatcher, Spectral sequences in algebraic topology, available at http://www.math.cornell.edu/ hatcher/SSAT/SSATpage.html.

[43] O. Aharony, A. Hashimoto, S. Hirano and P. Ouyang, D-brane charges in gravitational duals of $2+1$ dimensional gauge theories and duality cascades, JHEP 01 (2010) 072 [arXiv:0906.2390] [INSPIRE].

[44] A. Hanany and B. Kol, On orientifolds, discrete torsion, branes and M-theory, JHEP 06 (2000) 013 [hep-th/0003025] [INSPIRE].

[45] A. Hanany and J. Troost, Orientifold planes, affine algebras and magnetic monopoles, JHEP 08 (2001) 021 [hep-th/0107153] [INSPIRE].

[46] C. Beem, L. Rastelli and B.C. van Rees, The $\mathcal{N}=4$ superconformal bootstrap, Phys. Rev. Lett. 111 (2013) 071601 [arXiv: 1304.1803] [INSPIRE].

[47] P. Liendo, I. Ramirez and J. Seo, Stress-tensor OPE in $\mathcal{N}=2$ superconformal theories, JHEP 02 (2016) 019 [arXiv: 1509.00033] [inSPIRE].

[48] P.C. Argyres, A. Kapustin and N. Seiberg, On S-duality for non-simply-laced gauge groups, JHEP 06 (2006) 043 [hep-th/0603048] [INSPIRE]. 